\title{
Designing Self-directed Learning in Mathematics for High Ability Learners in Dunman High School, Singapore
}

\author{
Geok Hwa Khoo \\ Dunman High School \\ Singapore
}

\begin{abstract}
This paper presents the exploration of mathematical applications and self-assessment strategies to help students learn mathematical graphs. Students make use of mathematical software or tools in the discovery of different types of graphs, making observations and generalisations of these characteristics themselves rather than being taught information on the graphs by their teachers.

The lesson package to achieve this also involves helping students appreciate the relevance of graphs through a Mathematical Modelling task. The task requires students to research for graphs used in solving real-life context that resemble the basic graphs that they have learnt, explain the properties of the graphs and draw the relevance of the characteristics of the graph in the context. This allows students to appreciate practical application of graphs in real life.

In addition, the paper advocates incorporating strategies like self-assessment and self-reflection to develop students to become more self-directed in learning Mathematics.
\end{abstract}

\section{Introduction}

Founded in 1956, Dunman High School (DHS) is an autonomous co-educational secondary school in Singapore offering the Integrated Programme. For years, the school has established a name as a premier school which nurtures leader who are not just honorable but loyal citizens as well.

In 2018, DHS has a student population of about 2300 distributed across six levels: Years 1 to 4 (Junior High) and Years 5 and 6 (Senior High). Student ratio of boys to girls is about 2:3. Students admitted to DHS are the top 5-8\% of the nation in their Primary School Leaving Examination. Hence the school takes concerted efforts in providing quality curriculum for these high ability learners.

Typically, secondary schools in Singapore teach students how to sketch different types of graphs in a didactic manner with the teacher demonstrating each type of graph, and describes each graph's characteristics. Then, students are tested to see if they are able to reproduce, for instance, graphs of $y=1 / x$ or $y=x^{2}+1$. Often, students find it difficult to understand the various types of graphs and are disinterested to sketch the graphs without meaningful context.

This paper aims to present the method and lesson package used to teach Graphs of Functions to Year Three (age 15) high ability learners in DHS that promotes self-directed learning. The lesson package incorporates developing $21^{\text {st }}$ Century Competencies like the use of Information and Communication Technology (ICT), Mathematical Modelling (MM), viva voce (oral presentations), self-assessment and self-reflection.

There is a growing emphasis over the last decade on the use of Mathematical Modelling (MM) tasks in the teaching of Mathematics, DHS incorporates MM in its levels of Years 1 to 4. Many of the MM tasks set are done in groups outside the classrooms. Another growing trend is to include Problem in Real-world Context (PRWC) in the high-stake examinations. This requires students to solve problems integrating different concepts and skills in Mathematics to solve some common problems in life, e.g., calculating postage cost for mailing parcels. Schools and the examination board have placed greater emphasis on the students' ability to apply knowledge and skills in real-life tasks in their daily lesson as well as in the high-stake examinations. For the high ability learners in DHS, both MM and PRWC are becoming essential components in the teaching and learning of mathematics. 


\section{Rationale}

The topic on Graphs of functions is a very interesting and practical one. Graphs appear frequently in our daily lives and are often used in other subjects such as Physics, Geography and Economics, besides Mathematics. Berg and Smith [1] opines that students "do not have the mental tools to engage in a high-level construction or interpretation of graphs", and they find it challenging to sketch the rather abstract graphs. Hence, Mathematics teachers need to look for ways to aid students in gaining competence in this area. Graphs of functions is usually taught at Secondary 3.

In terms of pedagogy, Self-directed learning is emphasised in DHS as the students are of high ability and they are motivated in learning and constantly seeking improvement. The school continually seeks innovative and effective ways in teaching and learning to provide a quality curriculum for deep learning. One of the areas of growth identified is motivating our students to look beyond test results and be more self-directed in their learning as $21^{\text {st }}$ century learners. Use of mathematical tools in learning graphs and appreciating beauty of such graphs is an innovative change in the pedagogy here as it rides on the IT-savvy age of the students, taps on students' interest and learning profile to make discovery and construct knowledge themselves. Hence, self-directed learning of Mathematics is essential to develop selfregulating learners who could monitor their own thinking and reasoning and are able to see applications and modeling of mathematics concepts. This is aligned to the Singapore Mathematics Framework. (see Figure 1).

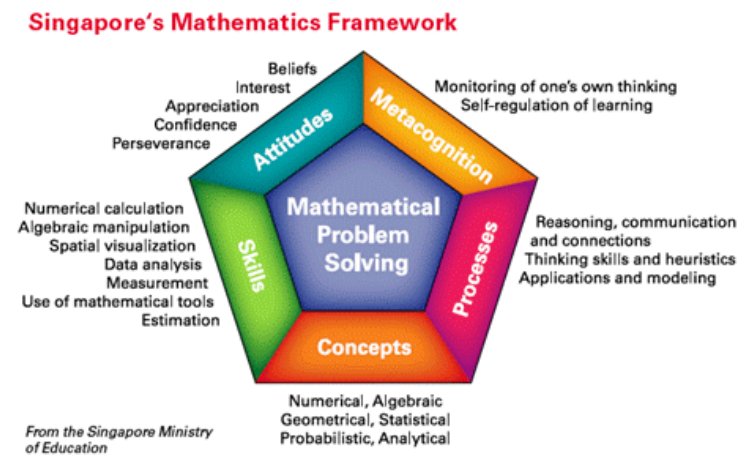

Figure 1. Singapore Mathematics Framework [4]

Furthermore, embracing the Singapore Ministry of Education (MOE) philosophy ${ }^{1}$ that assessment is integral to, and shapes, students' learning, the school has been working to strengthen our formative

\footnotetext{
${ }^{1}$ MOE Assessment Philosophy (2012) states that "Assessment is integral to learning"; "Assessment begins with clarity of purpose, and a balanced assessment system has both Assessment of
}

assessment strategies in a school-wide approach to enhance learning. Self- Assessment and Selfreflection are two such effective strategies as Assessment for Learning (AfL).

\section{Literature Review}

Ample research in the everyday use of mathematics shows that not only is test performance different when the contexts of testing are changed, but also (and because of the change in context) mathematical performance cannot be isolated as cognitive ability in people's heads Lave [4]; Scribner [6]. Rather, "cognition" is situated such that the setting contributes to and constitutes an important structuring device for framing problems and constructing solutions. Supported by these findings, this paper highlights on student engagement, via the first phase of Mathematical Modelling, i.e. to understand the problem in the real-world scenario and to make meaning of the graphs they have learnt. The MM learning task challenges the students to provide an abstraction that reduces a problem to its essential characteristics fitting a mathematics model.

Mathematical models are useful for a variety of reasons. Foremost, models represent the mathematical core of a situation without extraneous information. The equation $a=b+6$, for instance, removes all of the unnecessary words from the original statement "Alison is 6 years older than Brenda." In addition, people can use models to explore various scenarios cost-effectively. A jet engine manufacturer would prefer to design a mathematical model and conduct simulations on a computer, rather than incur the costs of building engines for testing purposes.

On assessment for learning, Black and Wiliam's research [11] highlights the student as the main decision maker in improving achievement. Sadler [10] adds that achievement hinges on developing students' capacity to monitor the quality of their own work during production.

Chappuis [2] opines that students need to know where they are and what their learning goals are, before they could find ways to reach the goals. According to Chappuis [2], examples of assessment for learning strategies include using examples and models of strong and weak work, offering descriptive feedback, getting students to do self-assessment and goal setting, engaging students in self-reflection and keeping track of their learning. Self-assessment would help students to take learning on their own instead of relying on the teachers or external parties to direct them.

Learning and Assessment for Learning" and "Assessment should gather information to inform future practices". 
Self-Assessment and Self-Reflection are formative assessment practices designed to meet students' information needs to maximise motivation and achievement, by involving students from the start in their own learning [9]. It is important to develop students' capacity to monitor the quality of their own work during production, to ask themselves "Where am I going? Where am I now? How can I close the gap?"

\section{Methodology}

This self-directed learning of graphs involves exploration of mathematical apps and tools to provide opportunities for students to discover the beauty of mathematical graphs. Students make use of software or apps in the discovery and make observations of the characteristics of the graphs themselves.

Phase 1: Self-directed Learning on Basic Graphs in Powers Functions and Exponential Functions. (Appendix 1_Part 1 worksheet)

Students download the graphing software into their phones or tablets, and follow a guided worksheet to explore and learn the shapes and features of the different types of basic graphs e.g. exponential, power functions, etc.

Extract of the Lesson Plan for Phase 1 is as below: Instructions to students

1. You can use either (i) Graphmatica (PC) or (ii) Desmos Graphing Calculator (Android/iOS) to explore the various graphs of functions in this section.

2. Graphmatica

- Go to http://www.graphmatica.com/

- Download Graphmatica 2.4 for Windows or Mac OS X and install in your PC or Laptop.

3. Desmos Graphing Calculator

- Go to 'Google Play Store' or 'Apple App Store' and search for 'Desmos Graphing Calculator'

- Download and install the app in your hand phone/tablet.

4. You will need a pencil, an eraser and a pen to complete this part.

5. Approximate time needed for this part: 45 minutes

When exploring the various graphs, look out for the following points:

- General shape of the graph

- Tail end-behaviour of the graph - how does $x$ and $y$ change correspondingly as the other variable tends to positive or negative infinity?

- Values of $x$ for which the function is defined/undefined (if applicable). How does $y$ change for such values of $x$ ?

- $\quad y$ - and/or $x$-intercept(s), if any.

\section{$\$ 1 \quad$ Power Functions}

Power functions are a broad class of functions of the form $y=a x^{n}$ where $a$ is non-zero and $n$ is any real number. In this chapter, we shall only look at the cases where $n=-2,-1,0,1,2,3, \frac{1}{2}, \frac{1}{3}$.

For each case, we will observe the basic graph where $a=1$.

For each case, we will observe the basic graph where $a=1$.

(a) $n=\mathbf{0}$

Graph: $y=$

Without using any graphing software, sketch the graph in the axes below.

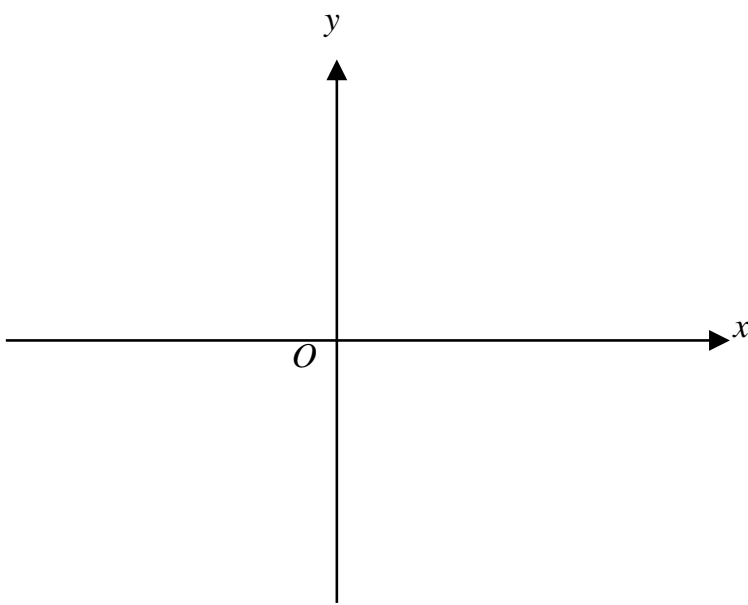

- $\quad$ The graph is a line.

- Axial-intercepts ('Nil' if doesn't exist):

$x$-intercepts: $x=$

$y$-intercepts: $y=$

(b) $n=1$

Graph: $y=$

Without using any graphing software, sketch the graph in the axes below.

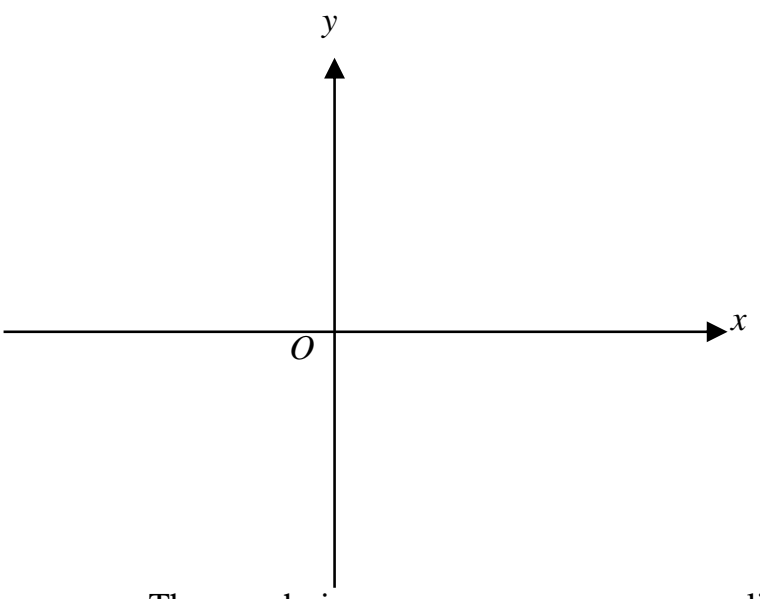

- $\quad$ The graph is a line passing through the

- Axial-intercepts:

$x$-intercepts: $x=$ 
$y$-intercepts: $y=$

- As $x$ becomes positively large, $y$ becomes positively large.

(Notation: As $x \rightarrow+\infty, y \rightarrow+\infty$ )

As $x$ becomes negatively large, $y$ becomes negatively large.

(Notation: As $x \rightarrow-\infty, y \rightarrow-\infty$ )

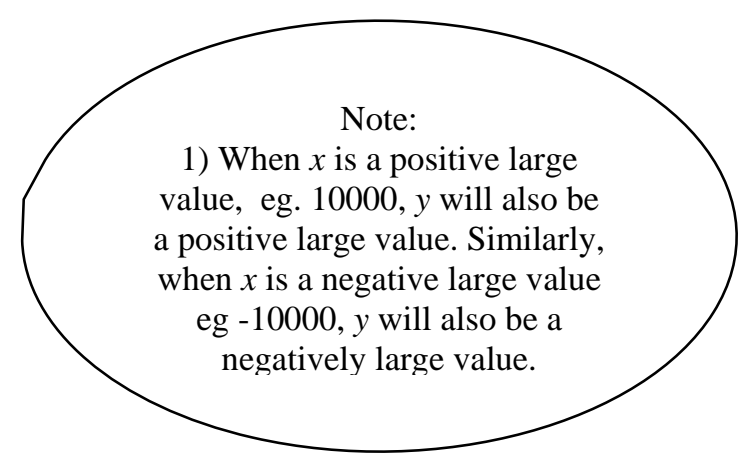

(c) $n=2$

Graph: $y=$

Without using any graphing software, sketch the graph in the axes below.

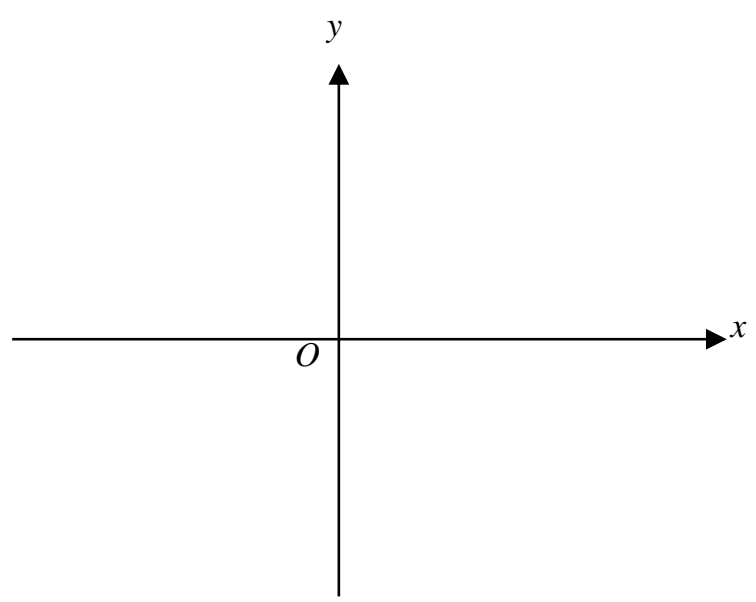

- The graph is a curve passing through the

- Axial-intercepts:

$x$-intercepts: $x=$ ; $y$-intercepts: $y=$

- $\quad$ As $x$ becomes positively large, $y$ becomes

As $x$ becomes negatively large, $y$ becomes

This is known as the tail-end behaviour of the graph.

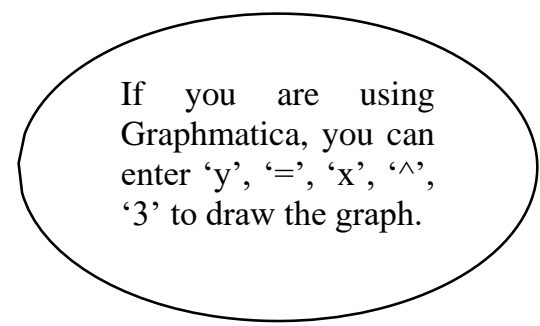

(d) $n=3$

Graph: $y=x^{3}$

Using any of the graphing software, sketch the graph in the axes below.

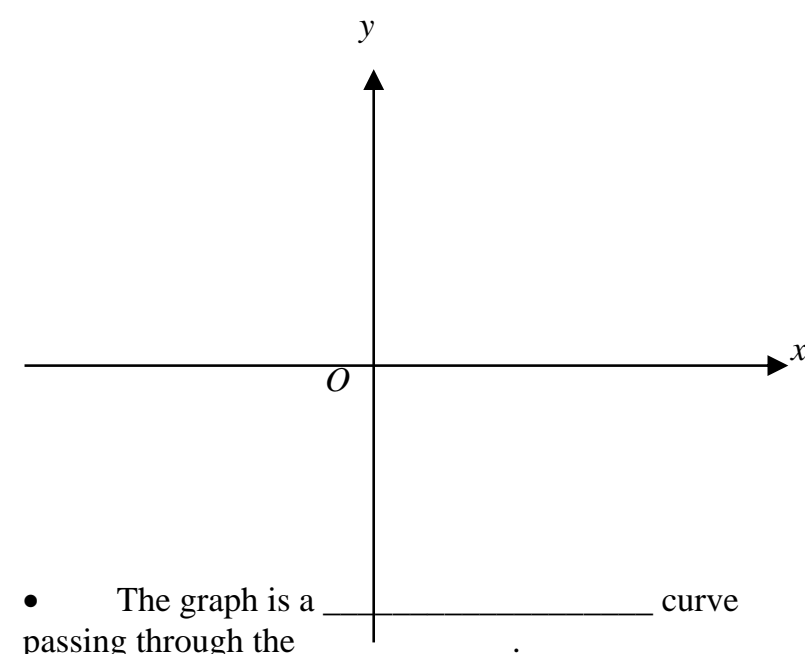

passing through the

- Axial-intercepts:

$x$-intercepts: $x=$ ; $y$-intercepts: $y=$

- As $x$ becomes positively large, $y$ becomes

As $x$ becomes negatively large, $y$ becomes

- What do you think is the gradient at of the curve at $x=0$ ?

$(0,0)$ is known as a stationary point of inflexion. You will learn more about this in Year 4.

Phase 2: Application of graphs to real life via Mathematical Modeling Task.

Students research on real-world scenarios where the graphs encountered in their Student Worksheet are observed. They present one such scenario and explain the assumptions used in the scenario for the modeling and propose alternative choice of graph that might model the scenario better. They are asked to defend their choice of graphs through classroom discussion and debate. 


\section{Process of Mathematical Modelling}

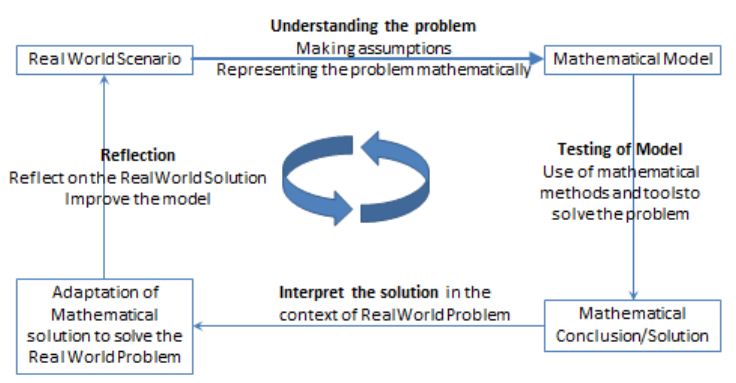

Figure 2. Process of Mathematical Modelling [8]

The Lesson Plan for Phase 2 is as below:

Instructions to students:

Having learnt about a number of graphs in Part 1, we shall now think about how any real-world scenarios can be modelled by a mathematical model, which can be in the form of a graph. This process constitutes Stage 1 of Mathematical Modelling, i.e. design a mathematical model from a real-world scenario, after simplifying the problem by making assumptions about the multiple factors affecting the issue.

Take a look at the example shown on the next page before working on the Mathematical Modelling task provided.

\section{Example of a Mathematical Modelling (Stage 1) Population Growth_(Adapted from [5])}

\section{Section 1}

Describe a real-world scenario

Population growth is known as one of the driving forces behind environmental problems, this is due to the fact that the growing population demands increasingly more (non-renewable) resources for its own application. Growth is usually thought of as a linear process: an increase by a constant amount over a period of time. The new amount is not influenced by the amount already present. For exponential growth, this is different, because the increase of a factor is proportional to what is already present. In terms of population growth, the numbers of people already present always influences the number of children born in any country. It is however not a simple matter of a constant doubling of the amount. Other factors, such as fertility and mortality rates, influence population growth, and the sexes and age of people already present, and rational decisions influence whether or not people will actually have one or more children.

\section{Section 2}

Sketch of graph to model real-world scenario

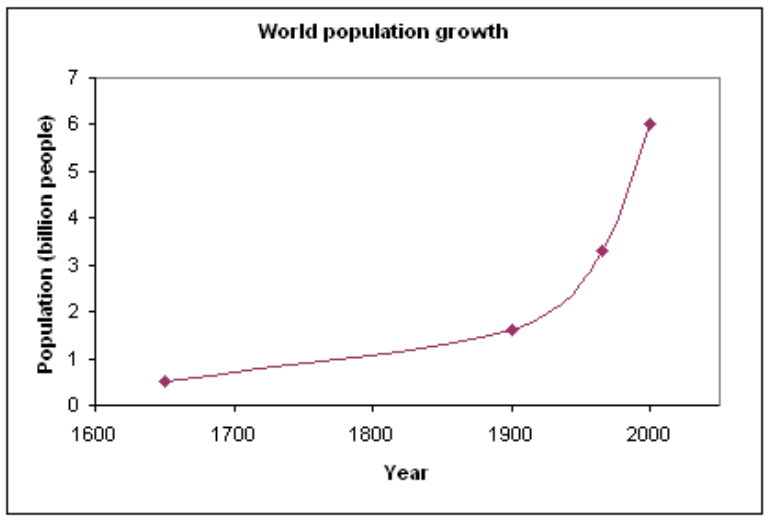

Figure 3. World Population Growth [5]

\section{Section 3}

Explanation of graph with relevance to influencing factors

In 1650 , the count for human population was only 0.5 billion heads. By 1900, the population had increased to 1.6 billion heads and was growing increasingly more rapidly, to 3.3 billion in 1965 (see graph above). In addition to the population growing, the doubling time was also decreasing, which indicated that the rate of growth increasing. This rapid growth increase was mainly caused by a decreasing death rate (more rapidly than birth rate), and particularly an increase in average human age. By 2000 the population was 6 billion heads, however, population growth (doubling time) started to decline after 1965 because of decreasing birth rates.

\section{Mathematical Modeling Task Instruction to students: Section 1}

Research about a real-world scenario (excluding population growth) and describe the scenario as clearly and concisely as possible.

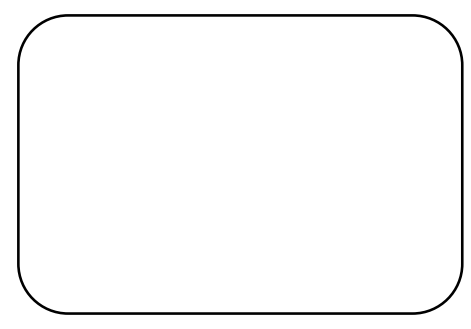

\section{Section 2}

Select a graph from Part 1 notes which models the trend of the real-world scenario described in Section 1 and indicate both horizontal and vertical axes with the relevant descriptions. 


\section{Section 3}

Describe the trend of the graph and explain, with reference to the relevant factors influencing the realworld scenario, how the factors affect the trend of the graph.

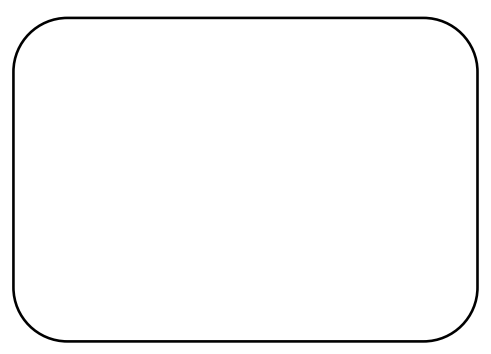

Phase 3: Transformation of Graphs Reflections, Vertical and Horizontal Translation

Students are given a second guided worksheet to complete in a class activity on transformation of graphs. They are to observe and explain the effects of the transformation techniques on the graphs of $y=\mathrm{f}$ (x) for

\section{i. Reflection}

$$
\begin{aligned}
& y=-\mathrm{f}(x) \\
& y=\mathrm{f}(-x)
\end{aligned}
$$

\section{ii. Translation}

$$
\begin{aligned}
& y=\mathrm{f}(x) \pm k, k>0 \\
& y=\mathrm{f}(x \pm k), k>0
\end{aligned}
$$

They have to plot and compare different graphs, observe trends, and deduce the different transformations that the graphs undergo through answering questions that require critical thinking. These are designed to assist students to co-construct knowledge to arrive at the right conclusions on the transformation after each activity

\section{Phase 4: Assignment on Part 1 \& 2}

After lesson consolidation, an assignment will be administered to assess their learning. Based on the assignment, students are given a first round of feedback based on the quality of their answers.

\section{Phase 5: Self-assessment and self-reflection}

Teaching students to self-assess and set goals; and engaging students in self-reflection and letting them keep track of and share their learning are essential in developing self-directed learners. To help students develop self-assessment strategies, there are three broad components, namely self-assessment, justification and goal-setting. First, students make a judgment about what they know, have learnt or have mastered in self-assessment. This is followed by justification where they attempt to show evidence in their work to support their judgment. Finally, students embark on goal-setting to make sustainable and practical plans for their future learning. It is to be noted that all these three components need not be present at the same time during the application of the strategy.

Figure 4 below illustrates how students assess themselves via the use of a rating scale, using a selfassessment check-list of the topic of graphs to help learners identify the area or concept that need clarification and what other topics.

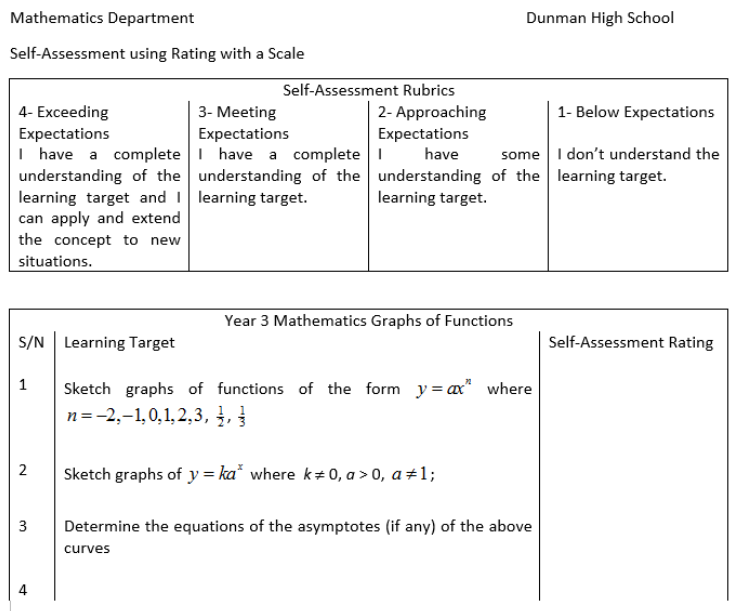

Figure 4. Self-assessment Using Rating with a Scale

After the completion of a topic, and before it is tested, students are given reflection forms with the learning targets and self-assessment rubrics for them to assess their current level of mastery. Students who perceive themselves to be lacking in any particular learning outcome are encouraged to review these specific areas and follow up with their teachers. This student-centric practice enables students to develop initiative and responsibility, as well as enhance their self-regulatory abilities.

To provide justification and evidence for their self-assessment, students are asked to review a list of possible reasons to justify their self-assessment and then identify those which are applicable to them (see Figure 5).

REFLECTING
I could not answer / answer the question wrongly
because I ...
did not understand this topic
could not remember what I have learnt
could not apply what I have learnt
applied the idea wrongly
had some missing key words
phrased my answer poorly leading to misconception
was careless
did not show working where required
did not understand what the command word in the
question requires; eg explain, describe, etc
did not understand the question because of the
language used
did not manage my time well during test
Others:

Figure 5. Justification in self-assessment 
Phase 6: Level Quiz \& EOY Examination

The Level Quiz for the 400 Year 3 students is used to measure learning of graphs in a self-directed manner. Question on graphs is set in End-of-Year (EOY) examination to assess the retention of knowledge and skills and the effectiveness of the methodology. Both Level Quiz and EOY examination question yield results to be collated for analysis and follow-up.

\section{Teacher collaboration in the Lesson design}

In this research study in 2016, the five teachers teaching the Year 3 students came together as a Professional Learning Team to design the lesson package on the topic. It is through the collaborative work and sharing of resources that culminated in the lesson package for the whole level of Year 3 students.

To promote critical thinking, special care was taken in the development of the worksheets with probing and thinking questions inserted after each activity to assist the students to come to the right conclusions and achieve the intended learning outcomes. This took up more effort and time with the team since the first two parts of the chapter is to be done on their own (HBL) by the students. To ensure that learning has taken place, corresponding level quiz questions were set.

After implementing the self-directed learning package, the teachers did a quick review in their respective classes and collected feedback from their students. Finally, a survey was designed for all the teacher implementers to evaluate their own learning via this Professional Learning Team project.

\section{Findings}

Based on the research study done with High Ability Learners in DHS in 2016, where students underwent Phase 1 to 4 and 6, data were collected to study the effect of the self-directed learning on students' results at tests.

After the students have gone through the comprehensive Part 1 and the Mathematical Modelling research, students presented their research and explained the trend and the shape of the graphs as applications in the real-world context. Based on the students' work, it was found that they have acquired the ideas of how graphs could be relevant and applied in the real-world scenario. They were also able to list out factors that determined and influenced the trends and shapes of the graphs. Last but not least, they were able to communicate these factors using Mathematical language in the form of Viva Voce mode.
The Level Quiz score was used to validate the effectiveness of the self- directed learning package. For the Level Quiz, students were given three types of questions on the topic, Graph of Functions. The performance of the students is given below. Based on the facility indexes, it was concluded that majority of the students were competent in sketching the transformed basic graphs and they were able to interpret the graphs to state the given equations. For Question 2, it was testing on whether students were able to transform a given graph where the equation is not given. They would have to understand and apply the concepts of transformation. This question posed a challenge to them as there were many key features given in the original graph which they would need to know how to address each feature under the transformations.

Table 1. Mathematics 1 Level Test Item Analysis

\begin{tabular}{|l|l|l|l|}
\hline Question & $\begin{array}{l}\text { Average } \\
\text { Performance }\end{array}$ & Total Marks & $\begin{array}{l}\text { Facility } \\
\text { Index }\end{array}$ \\
\hline 1 & 2.06 & 3 & 0.69 \\
\hline 2 & 2.02 & 3 & 0.67 \\
\hline 3 & 3.90 & 5 & 0.78 \\
\hline
\end{tabular}

Another check point for the effectiveness of the lesson is the end of year examination where a slightly more challenging question is given to the students. Table 2 shows that the targeted question had a facility index of 0.71 , signifying that the students have performed beyond expectation, which indicated that students were able to consolidate the learning of this chapter at the end of the year.

Table 2. Mathematics 1 EOY Examination Item Analysis

\begin{tabular}{|l|l|l|l|}
\hline Question & $\begin{array}{l}\text { Average } \\
\text { Performance }\end{array}$ & Total Marks & Facility Index \\
\hline 7 & 6.37 & 9 & 0.71 \\
\hline
\end{tabular}

Qualitative data was also studied at these two assessments to determine whether students have grasped a strong understanding of the topic.

Furthermore, students' reflection showed that the topic taught in this manner was refreshing due to the different nature that the chapter was introduced to them. Instead of routine classroom teaching, students felt that the experiential approach involving the use of the graphing app in their phone was exciting and fun. 
In addition, having an easy access to a selfchecking software helped them to feel more confident in their learning and this translated to their motivation to want to do more questions.

Students also felt that they were able to regulate their own learning pace since the notes were designed to allow for self-directed learning. As such, there was little pressure to "keep up" with a classroom pace and there was sufficient time for practice.

The opportunity to explore real-world applications and predict an appropriate equation to model the trend allowed students to see purpose and relevance in this topic. They also felt that the discussions on assumptions made when predicting equations were meaningful and served to remind them of the importance of the human element in intelligence.

\section{Teacher Reflections}

Based on the survey results and discussion, the teachers reflected that the experience of working together to craft the teaching package was very satisfying be it in trying to come up with the worksheets or the Mathematical modelling task. Having to guide the students when they shared about their MM tasks also opened new doors for some of them since this is the first such task for them. Together, they grew professionally.

Teachers realized that to craft a comprehensive self-directed learning package, they need to consider various factors. Firstly, they need to be aware of the prerequisites of the chapter and ensure that the students are equipped to learn the new chapter on their own. If not, students will find it difficult right at the beginning.

Secondly, as fast learners tend to have shorter attention span, they need more variety in the work that they are given. For the chapter on Graphs of Functions, the teachers harness on app-based ICT in getting the students to identify the shape and equations of the basic graph. Students are also required to research online for an application on the basic graph that they have learnt. After which, they consolidate their learning by drawing a few transformed graphs. By giving students a variety of tasks, the team of teachers hope to use a multifaceted approach in engaging this generation.

Thirdly, the team also reflected that they would need to have a more structured approach in consolidating the self-directed learning. For example, they could use an approach called team-based learning in assessing how much the students have learnt on their own. Alternatively, they could harness on ICT to check on the understanding of the students before moving on to the more difficult segment of the chapter.

\section{Conclusion}

Use of strategies on self-directed learning, selfassessment and self-reflections, coupled with mathematical tools and real-world application, help high ability students in DHS to learn and retain concepts of mathematical graphs well. It develops self-regulatory qualities of these motivated learners to thrive in the $21^{\text {st }}$ Century.

More research can be done to examine the effectiveness of using self-directed learning through Math Modelling and the use of ICT to other topics in Mathematics and other ages of students. For instance, the team could examine whether these strategies could help lower secondary students gain a deeper understanding of topics that require visualization, like linear graphs.

More work is needed to make self-directed learning a prevalent pedagogy for high ability learners in DHS to challenge them cognitively and socially and instill in them more joy in learning Mathematics.

\section{References}

[1] Berg, C. A., \& Smith, P. (1994). "Assessing students' abilities to construct and interpret line graphs: Disparities between multiple-choice and free-response instruments", Science Education, 78, pp. 527-554.

[2] Chappuis, J. (2009). Seven Strategies of Assessment for leaning. Person Education. MA.

[3] Khoo, G. H., Low, C., Leong, F.L., Koh L.K, Phua, P. L Siah, S. Y. (20116) Learning to Assess, Assess for Learning. In G.H. Khoo \& H. Galistan (Eds), The Art \& Craft of Teaching (Vol 2, pp. 162-170). East Zone Centre of Excellence for Teaching \& Learning. Singapore.

[4] Lave, J. (1988). Cognition in practice: Mind, mathematics and culture in everyday life. Cambridge University Press, Cambridge, 1993.

[5] Lenntech. (2016). Population Growth as a Driving Force for Environmental Problems. Retrieved from http://www.lenntech.com/population-growth-andenvironment.htm\#ixzz49SgjT11J

[6] Scribner, S. (1986). Thinking in action: some characteristics of practical thought. In R. J. Sternberg \& R. K. Wagner (Eds.), Practical intelligence: Nature and origins of competence in the everyday world. Cambridge University Press, Cambridge, pp. 13-30.

[7] Singapore Ministry of Education (Curriculum Planning \& Development Division. (2012). Primary Mathematics Teaching and Learning Syllabus. Ministry of Education, Singapore. 
[8] Singapore Ministry of Education. (2013). Secondary mathematics syllabuses. Singapore: Author. Retrieved form http://www.moe.gov.sg/education/syllabuses/sciences/

[9] Stiggins, R., Arter, J., Chappuis, J. \& Chappuis, S. (2004). Classroom assessment for student learning: Doing it right- using it well. ETS Assessment Training Institute. Portland, OR.

[10] Sadler, D.R. (1989) 'Formative assessment and the design of instructional systems', Instructional Science 18: $119-44$.

[11] Black, P., D. Wiliam, D., (1998a), "Assessment and Classroom Learning", Assessment in Education, Vol. 5, pp. 7-71. 